\title{
Twinkle, twinkle
}

How astronomers investigate alien planets

The NASA Kepler Space understanding of the universe. By continuously observing over half a million stars during its four year mission, Kepler discovered thousands of exoplanets. Many hundreds of these are Earth-like of them reside in the habitable 'Goldilocks' zone Kepler and similar space-based telescopes like TESS discover planets purely by infererence, never actually 'seeing' these planets. Instead they watch for tell-tale periodic dips in brightness. When a star twinkles, suggesting an alien Steve B. Howell at NASA's Ames Research Centre has the jus determining what it is. Using some of the largest groundbased telescopes on Earth and a technique called speckle interferometry, the researchers are identifying the planets which might be best-suited to harbouring life. o you believe in aliens? More
than one in two people in the
UK, Germany and the US believe there is intelligent life out there in the universe, meaning that - whether your
belief is rooted in crop circles, statistic belief is rooted in crop circles, statistics or gut feeling - 'you are not alone'

100 years ago the hunt for alien life was limited to speculations about Mars and field no Nowas we can look funter our own solar system: exoplanets.

Unquestionably, the game-changer in the field of exoplanets was the Kepler space telescope. Johnannes Kepler (1571-1630) was a mathematician astrologer, and astronomer. He used observations of the planets in our solar system to develop his famous laws of planetary motion. Kepler also worked on optics and invented an improved version of Galileo's astronomical telescope - a refracting type or a Keplerian telescope.

Launched in 2009, armed with photometer as its only scientific began surveying one field of velescope sky. Kepler discovered more exoplanets

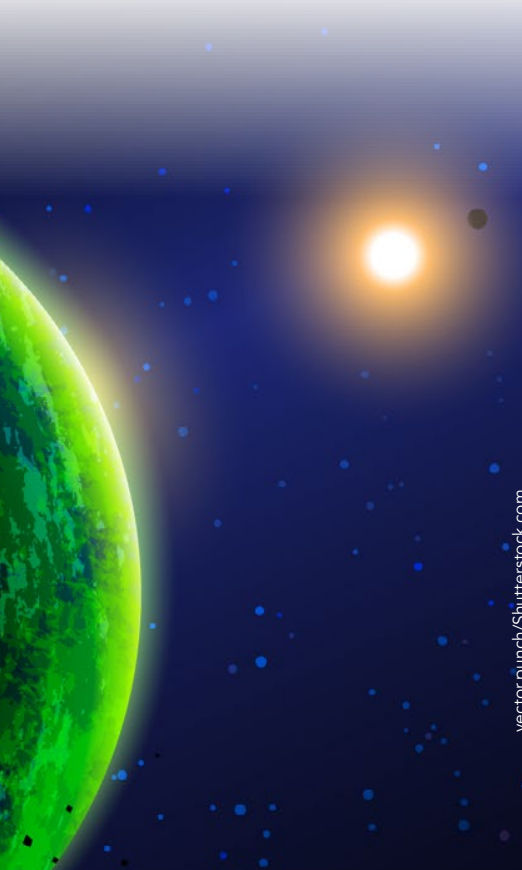

during its mission than all other discovery methods combined.

Of particular interest to Kepler were discoveries of Earth-like planets. These 'rocky' planets have a similar size to Earth, and are within the 'habitable zone, usually defined as having a reside on the planet's surface.

\section{TWINKLE TWINKLE}

\section{SPOTTING EXOPLANETS}

Directly viewing exoplanets is not quite used photometric data to infer the existence of an exoplanet. This method is known as the transit method. It involves observing a star (or groups of stars) over time and monitoring their brightness very precisely. If an orbiting planet passes directly in front of - that is, it transits - a star, the light level will dip slightly and then return to normal. This will happen repeatedly, once for every orbit of the exoplanet. This is by no means the only way exoplanets can be detected. Another method, 'direct 'maging' looks at the thermal emission velocity method can identify exoplanethosting stars from the 'wobbe' as their planets orbit them. Finally, gravitational microlensing observes the lensing effect of gravity when a solar system passes directly behind another (unrelated) star.

None of these other methods, however, have discovered as many exoplanets as transit photometry. Over its years of service, the Kepler space telescope observed a staggering 530,506 stars every 30 minutes for four years and detected over 4,000 exoplanets. After two of its three reaction wheels failed in 2014, an ingenious proposal to repurpos Kepler as K2 led to an adaptation of spacecraft balance by using sunlight with

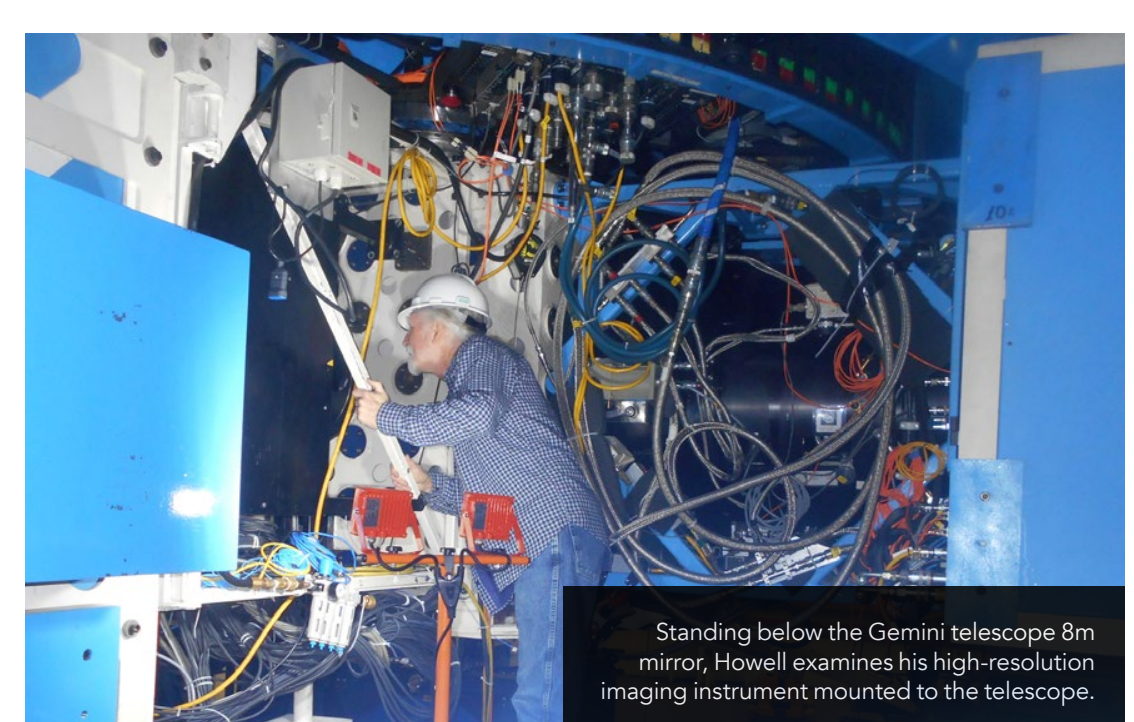

The K2 programme, with Dr Steve owell as lead scientist, gave new life to Kepler untilit retired in 2018 when 2 discovered many new exoplanets and extended the search for four additional years. The data collected by these missions is expected to continue revealing new exoplanets for several more years.

\section{THE NEED FOR}

XXPLANET FOLLOW-UP

A new wave of exoplanet space telescope missions is well underway, including NASA's

Transiting Exoplane Survey Satellite (TESS). TESS searches for transit signals across the
whole sky, and is whole sky, and is Unquestionably, the game-changer in the field of exoplanets was the Kepler space telescope. lso look for transits deeper in space than Kepler could. In addition, the James Webb Space Telescope and the Nancy Grace Roman Space Telescope will join the European Space Agency's CHEOPS, collecting information to help characterise existing exoplanets the search for life.

For each transit signal and new exoplanet discovered, it is important to perform detailed follow-up observation of the host stars. This crucial work has become Dr Steve Howell's domain.

Every star which shows a transit signature yields an exoplanet candidate,

time implying that a planet is regularly orbiting that star. The intrinsic brightness star's size: the amount the brightness dips is used to determine the relative size of the planet.

However, there are several reasons why the data might not give the full story. Especially problematic is the existence of binary star systems. This two star system affects the brightness readings in the data, and leads to incorre estimates of planetary size, density, and therefore possibilities of habitability. Dr Howell's research focuses in - quite which may orbit one star in a binary star . called high-resolution speckle imaging.

The need for this type of follow-up research was identified even before Kepler was launched. Howell has spent he last decade studying what he refers to as "transit-like" events. That is, since some of the exoplanet discoveries by transit observations may be false, the scrutinised in more detail.

Exoplanet transit searches from space elescopes such as Kepler or TESS, which gather information for many of the sky, have relatively low spatial resolution. Each pixel of their cameras may contain multiple stars, making it hard to identify which star is responsible for the observed transit-like regular dip in brightness.

To solve this problem, and to validate and characterise the exoplanets and their host stars, high spatial resolution images are required. Speckle imaging is a technique used by ground-based telescopes to

emove the

distortions caused by disturbances in the By re-constructing n image from

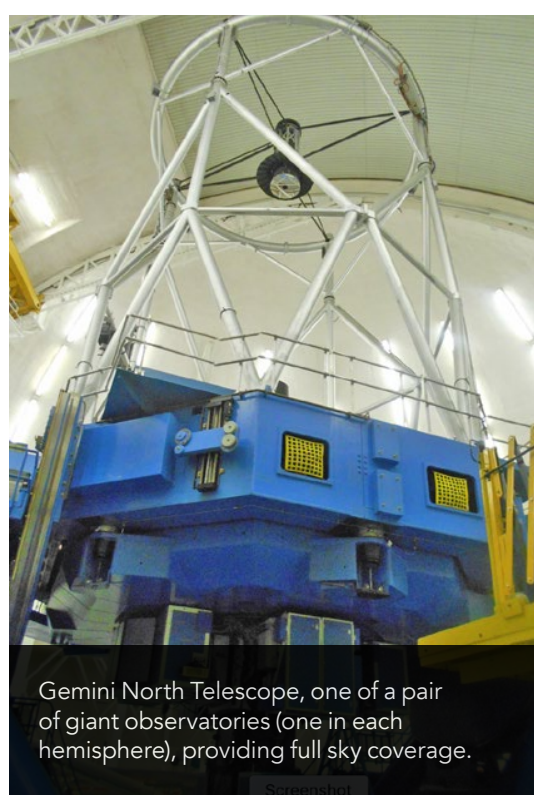

many short exposures with specialised software, very high-resolution images

\section{ULTRA-HIGH RESOLUTION}

As a result of Howell's research into ultra-high spatial resolution images, three new instruments have been built. These are installed at some of the largest ground-based telescopes in the produce the highest-resolution images yet achieved.

So far, the team has observed over 1,000 exoplanet hosting stars from Kepler and K2, and more than 500 published papers in the last year and a 


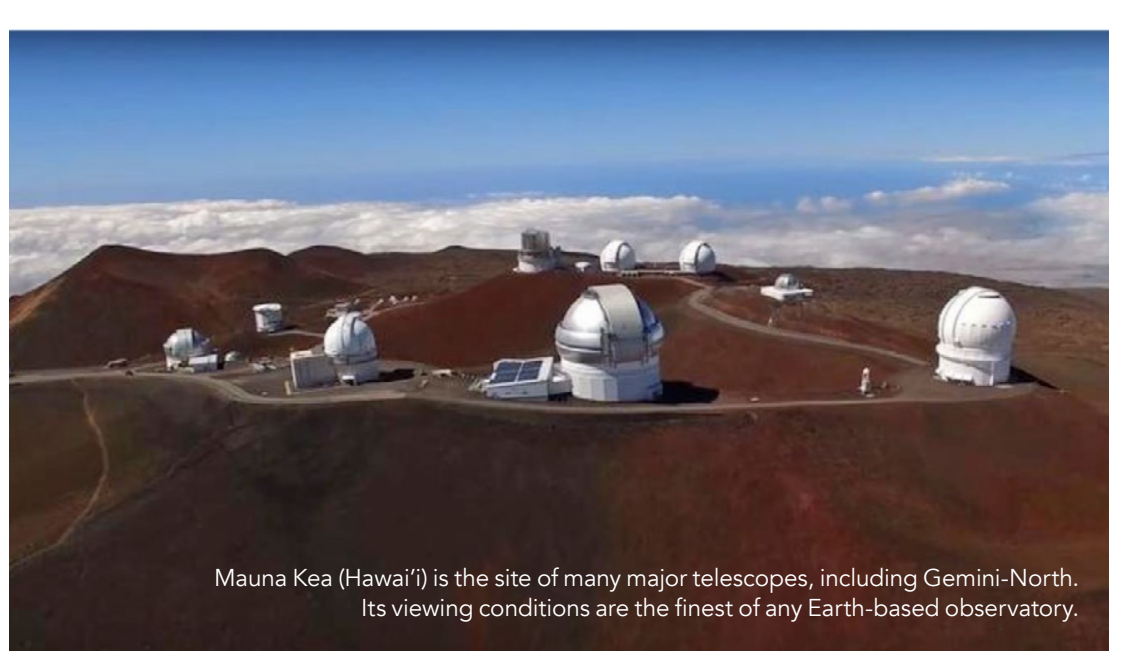

half alone. One of the most important learning points from this work is the value of community understanding of the host star or

\section{TWO STARS ARE BETTER THAN ONE}

(FOR PLANET FORMATION) exoplanet stars finds that almost half of all exoplanet-hosting stars are actually part of a binary or multiple star system. This has important implications for the way we interpret the transit signals. For example, 'contamination' light from the second star can reduce the dip in brightness when the exoplanet transits its host star.

The exoplanet will then be predicted to be smaller than it really is, meaning the planet is incorrectly characterised. What may look Earth-like from TESS Neptune-like ice giant

Recent research from Howell's team also details an interesting discrepan between binary star pairs with and without exoplanets. This was discovered in a survey of 186 exoplan host stars from the TESS mission.

\section{STELLAR WORK}

The most up-to-date estimates suggest average, 40 astronomical units from each other (the distance between our Sun an the planet Pluto). These estimates are based on observations of binary stars without regard to exoplanets. Howells survey of TESS data - looking specifict, at 45 binary systems with exo
provides a different result. part, and fall into a narrow range of possible inter-stellar distances.

Given that planets and their stars tend to be formed at the same time, Howell's planetary formation. His work suggests that many planets were formed under the same conditions which lead to binary star formation, a very different mechanism than our own single-star solar system

This isn't the only big discovery made through speckle imaging. Howell and his team also use their research to answer important questions about the exoplanets themselves. Which star in likely to orbit further from each other, the binary is the one hosting the planet?
typically around 100 astronomical units If two stars appear very close in space,

A new wave of exoplanet telescope missions is well underway, including Nasa's Transiting Exoplanet Survey Satellite (TESS) which searches for transit signals across the whole sky.

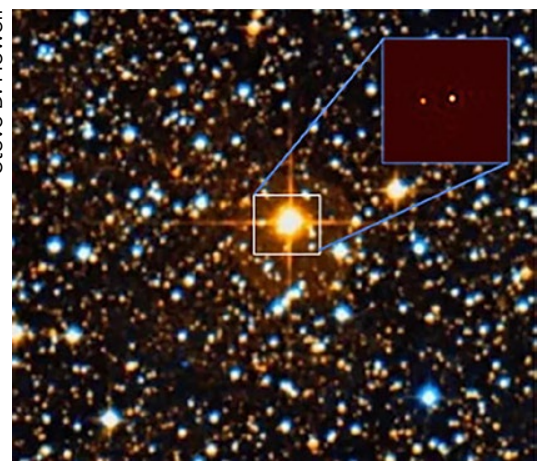
are they really close, or do they just Which planets are the best candidates for further investigation by telescopes that can perform spectroscopy to determine the composition of the exoplanet's atmosphere and search for signatures of life?

High-resolution speckle imaging gives us new ways of understanding exoplanets and their environments, Typical ground-based image of a star field,
showing a bright red star near the centre. A highresolution image of this star (inset) reveals that it including identifying those which might
is really a close binary pai-

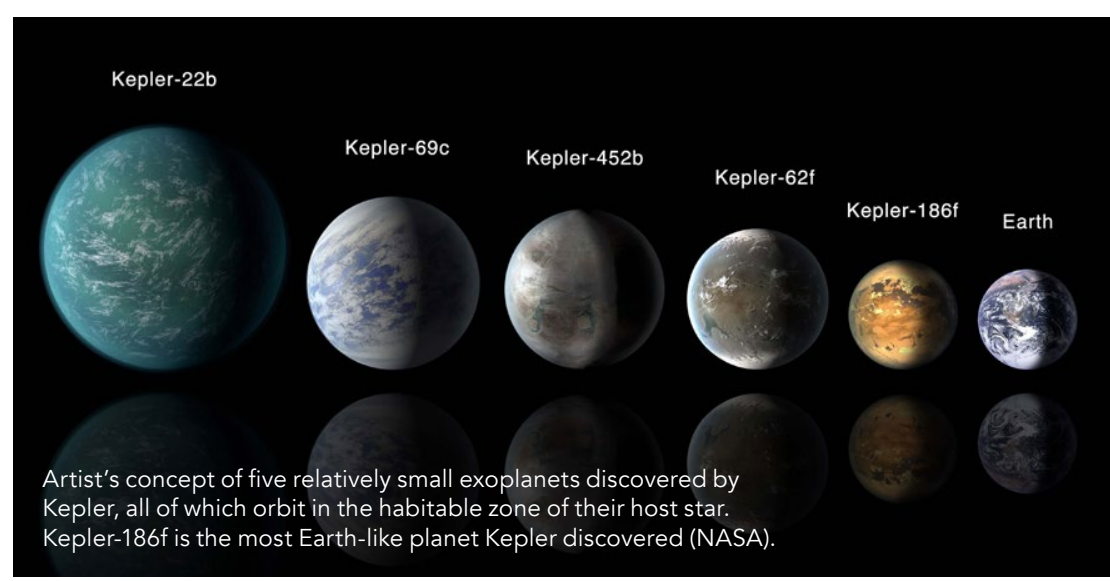

Research Objectives Steve Howell currently works to understand the characteristics and true nature of exoplanets - that is planets that orbit alien suns. Over 4,000 exoplanets are currently known, and Dr. How th sesearch aims dynamics and evolution. Making observations with new dynamics an evolution. Making observations with new on exoplanets, and in particular detailed study of the stars that they orbit.

\section{Detail}

Bio

Dr Steve B. Howell is a research scientist at NASA and was the project scientist for the Kepler and K2 missions. Steve has worked in many aspects of astronomy: pioneering the use of charge-coupled devices (CCDs), building innovative instruments, and involvement in many STEM activities. Howell has written over 800 scientific papers, nine books on astronomy, and two science fiction books. He enjoys scientific challenges, the great outdoors, vegetarian

gourmet cooking, and playing blues music.

Funding

NASA Exoplanet Program Office and NASA Headquarters

Collaborators - Crystal L. Gnilka - Kathryn V. Lester - Elise Furlan - Rachel A Matson - Mark E. Everett - Nicholas J. Scott

\section{NASA}

\section{References}

Howell SB, Scott NJ, Matson RA, Everett ME, Furlan E, Gnilka CL, Ciardi DR, Lester KV. (2021). The NASA HighResolution Speckle Interferometric Imaging Program: Validation and Characterization of Exoplanets and Their Stellar Hosts. Front. Astron. Space Sci. 8:635864. https://doi.org/10.3389/fspas.2021.635864 Howell SB, Matson RA, Ciardi DR, Everett ME, Livingston JH, Scott NJ, Horch EP, Winn JN. (2021). Speckle Observations of TESS Exoplanet Host Stars: Understanding the Binary Exoplanet Host Star Orbital Period Distribution. [Accepted, Astronomical Journal].

Howell SB, Sobeck C, Haas M, Still M, Barclay T, Mullally F, Troeltzsch J, Aigrain S, Bryson ST, Caldwell D. (2014). The K2 Mission: Characterization and Early Results. Publications of the Astronomical Society of the Pacific. 126398. doi: $10.1086 / 676406$.

\section{Personal Response}

Can you briefly detail the part speckle imaging has to play amongst the 'new generation' of space telescopes telescopes?

II One of the most fundamental questions of humanity is, Are we alone? Since humans looked up at the lights in the night sky the question of where we came from and who else is out there has been a fundamental driving force for exploration and discovery.

Speckle imaging and the research it enables is one aspect of the larger picture that someday will lead to
the discovery of life on an alien world. My current work provides detailed information on many exoplanets and the stars which they orbit in order to help all of us in astronomy produce alist of the best exoplanets to greatest opportunity for science to discover signatures greatest opportunity for science to discol
that life exists elsewhere in the Galaxy. 